\title{
Investigando a produção do conhecimento sobre a pessoa idosa longeva
}

\author{
Investigating the production of the knowledge on the long-lived elderly person \\ Investigando la producción del conocimiento sobre la persona mayor longeva
}

\author{
Talita Aquira dos Santos Lima', Tânia Maria de Oliva Menezes" \\ ' Universidade Federal da Bahia, Escola de Enfermagem, Núcleo de Estudos e Pesquisa do Idoso. \\ (Graduanda) Salvador-BA, Brasil. \\ " Universidade Federal da Bahia, Escola de Enfermagem, Núcleo de Estudos e Pesquisa do Idoso. Salvador-BA, Brasil.
}

Submissão: 04/02/2010 Revisão: 04/12/2010 Aprovação: 21/02/2011

\section{RESUMO}

Pesquisa bibliográfica, com abordagem quantitativa, que objetivou investigar a produção do conhecimento sobre a pessoa idosa longeva, nas bases de dados SCIELO e LILACS. Foram analisados oitenta e oito artigos científicos, dos quais 69,3\% foram produzidos no Brasil, concentrados na região Sudeste $(67,2 \%)$, com prevalência da abordagem qualitativa (77,3\%). Quanto à área de conhecimento, todas as pesquisas fazem parte da grande área Ciências da Saúde, com predomínio de Medicina $(76,1 \%)$. A enfermagem representou 4,5\% das produções. Os objetos mais estudados $(72,7 \%)$ foram casos clínicos ou relato de experiências. Conclui-se que a produção científica sobre o idoso longevo ainda é singela, mas relevante. Nesta ótica, torna-se essencial ampliar e qualificar as produções e os campos de conhecimentos direcionados a este universo temático.

Descritores: Idoso de 80 anos ou mais; Conhecimento; Envelhecimento demográfico; Longevidade.

ABSTRACT
This paper is a bibliographic review, with quantitative approach, that aimed to investigate the production of the knowledge on the long-lived elderly person, in the SCIELO and LILACS data bases. Eighty eight scientific papers were analyzed, from which $69,3 \%$ were produced in Brazil, concentrated in the Southeastern area $(67,2 \%)$, and with prevalence of the qualitative approach $(77,3 \%)$. As for the knowledge area, all the researches are part of the great area of Health Sciences, with prevalence of Medicine $(76,1 \%)$. Nursing contributed with $4,5 \%$ of the productions. The more studied objects $(72,7 \%)$ were clinical cases or report of experiences. One concluded that the scientific production on long-lived elderly person is still small, but relevant. In this optics, it is essential to increase and qualify the productions and the knowledge fields that address this thematic universe.

Key words: Aged 80 and over; Knowledge; Demographic aging; Seniority.

\section{RESUMEN}

Revisión bibliográfica, con acercamiento cuantitativo, que tuve como objetivo investigar la producción del conocimiento sobre la persona mayor de 80 anos, en las bases de datos SCIELO y LILACS. Ochenta ocho artículos científicos fueron analizados. Dentro estos, $69,3 \%$ se produjeron en Brasil, concentrados en el Sudeste $(67,2 \%)$, con el predominio del acercamiento cualitativo $(77,3 \%)$. En cuanto al área de conocimiento, todas las investigaciones son parte de la grande área de Ciencias de la Salud, con predominio de Medicina (76,1\%). La Enfermería representó 4,5\% de las producciones. Los objetos más estudiados $(72,7 \%)$ eran casos clínicos o relato de experiencias. Se concluye que la producción científica sobre el mayor de 80 anos es todavía aun simple, más relevante. En esta óptica, se considera esencial intensificar y calificar las producciones y los campos de conocimiento dirigidos a este universo temático.

Palavras clave: Anciano de 80 o más años; Conocimiento; Envejecimiento de la población; Longevidad. 


\section{INTRODUÇÃO}

A produção do conhecimento é fruto da dinâmica social, que considera, pois, a ordem do tempo e as mudanças ocorridas na sociedade. Deste modo, o conhecimento produzido deve demonstrar no decorrer dos anos um ritmo ajustado às exigências sociais e as necessidades de enfrentamento da população(1).

A nossa sociedade vem dando valor sobremaneira à investigação científica, como base para adoção ou não de determinadas ações, comportamentos e valores. Seguindo este caminho, a produção de conhecimento sobre o envelhecer tem ganhado uma atenção cada vez maior, fazendo circular o discurso dos experts e, por meio do vínculo da qualidade de vida na velhice, a certo tipo de práticas de sentir, pensar, falar e agir, definindo o campo como o da terceira idade ${ }^{(2)}$.

No Brasil, a pesquisa sobre envelhecimento é uma prática impulsionada a partir da década de 1980 . Existe um pequeno quantitativo de grupos de pesquisa especificamente destinados à investigação deste tema, cujos pesquisadores não apresentam titulação acadêmica elevada, fator que está estreitamente relacionado com a reduzida participação de discentes, mestres e doutores, nesses espaços. Além disso, existem poucos programas de pós-graduação stricto sensu relacionados especificamente para o estudo do envelhecimento - foram identificados três programas em Gerontologia, dos quais apenas um com doutorado; e três que contam com áreas afins a esta temática ${ }^{(3)}$.

O envelhecimento humano é um processo influenciado por múltiplos fatores determinantes: moleculares, celulares, sistêmicos, comportamentais, cognitivos e sociais. Estes interagem e regulam, desde o funcionamento típico, quanto o atípico da pessoa que envelhece ${ }^{(4)}$. Ademais, esses fatores também podem ser capazes de intervir na adaptação do indivíduo ao meio social em que vive, comprometendo a sua qualidade de vida ${ }^{(5)}$.

De acordo com a Organização Pan-Americana de Saúde, o envelhecer é um processo sequencial, individual, cumulativo, irreversível, universal, não patológico, de deterioração de um organismo maduro, próprio a todos os membros de uma espécie, de maneira que o tempo o torne menos capaz de fazer frente ao estresse do meio ambiente e, portanto, aumente sua possibilidade de morte ${ }^{(6)}$.

Sendo assim, o envelhecimento pode ser compreendido como senescência, ou seja, fase natural de alterações orgânicas, morfológicas e funcionais, próprias do avançar da idade, relacionadas ao envelhecer saudável, ao passo que, as alterações produzidas por condições de sobrecarga, afecções, estresse emocional, caracteriza a velhice marcada por condição patológica, denominado de senilidade ${ }^{(6)}$.

No Brasil, o envelhecimento demográfico tem como marco as mudanças nas taxas de fecundidade e mortalidade, com consequente aumento da expectativa de vida populacional, de forma rápida e desordenada. Esse processo teve início a partir de 1940, com o significativo declínio da taxa de mortalidade, o qual é prolongado até 1970 . No entanto, somente a partir da década de 60 , com a rápida e sustentada redução das taxas de fecundidade, dá-se início às modificações na estrutura etária no país, com procedente transição demográfica da população $0^{(7)}$.

Em conjunto com o aumento da expectativa de vida dos brasileiros ocorrem também alterações nos padrões de mortalidade e morbidade da população e, deste modo, remete a transição epidemiológica. Ao período caracterizado pelas doenças transmissíveis, atreladas às más condições de vida, pobreza, má nutrição, falta de higiene e saneamento básico, acrescentam-se as afecções crônico-degenerativas e as socioambientais, decorrente da degradação do ambiente, das condições de trabalho e das relações sociais através das drogas, violência e acidentes. Assim, convive-se em uma complexa realidade, na qual a violência coexiste com o recrudescimento de patologias transmissíveis, com ainda altos índices de mortalidade infantil e considerável aumento da expectativa de vida ${ }^{(8)}$.

O fenômeno do envelhecimento, hoje, é uma realidade mundial. Em todo o mundo, a proporção de pessoas com 60 anos ou mais está crescendo mais rapidamente que a de qualquer outra faixa etária. Entre 1970 e 2025, espera-se um crescimento de $223 \%$, ou, em torno de 694 milhões no número de pessoas mais velhas. Em 2025, haverá um total de aproximadamente 1,2 bilhões de pessoas com mais de 60 anos; até 2050, dois bilhões, sendo $80 \%$ nos países em desenvolvimento ${ }^{(9)}$.

É nesse contexto que os idosos em velhice avançada, idade igual ou maior que 80 anos, também vêm aumentando de forma proporcional e acelerada, tornando-se o segmento populacional com maior crescimento nos últimos anos, correspondendo a $12,8 \%$ da população idosa e $1,1 \%$ da população total|(6).

No que se refere à população brasileira, esta alcançará em 2050 o patamar de 81,29 anos de vida, aproximando-se assim, do mesmo nível atual de países como a Islândia $(81,80)$, Hong Kong, China $(82,20)$ e Japão $(82,60)$. Deste modo, observa-se "uma população que vem galgando degraus em sua longevidade" (10), já que a estimativa para 2050 é de 13,8 miIhões de brasileiros com 80 anos ou mais de idade ${ }^{(10)}$.

Assim, face ao envelhecimento populacional e ao impacto social viabilizado por este, tornam-se imprescindíveis novas concepções de atenção à população idosa e, sobretudo, aos longevos. Para tanto, é essencial fomentar as discussões e reflexões sobre o aumento da expectativa de vida por parte da sociedade em geral, e, mais especificamente, no meio acadêmico, do qual se espera maior produção de conhecimento sobre essa temática, especialmente, quanto à forma de proporcionar qualidade de vida às pessoas idosas ${ }^{(11)}$.

Diante do exposto, observa-se que o mundo caminha na perspectiva de uma população cada vez mais longeva, uma vez que os idosos com idade igual ou superior a 80 anos é a fração populacional que vêm crescendo significativamente a passos largos. Assim, no sentido de avaliar as implicações e relevâncias das investigações científicas relativas aos idosos longevos, e de contribuir para a ampliação de conhecimentos nesta área, elegeu-se como objetivo para este estudo o de investigar a produção do conhecimento sobre a pessoa idosa longeva na América Latina e Caribe. 


\section{METODOLOGIA}

Trata-se de uma pesquisa bibliográfica, com abordagem quantitativa, descritiva e exploratória realizada no período de agosto de 2008 a julho de 2009.

O estudo bibliográfico é desenvolvido com base em trabalhos já elaborados, incluindo principalmente livros e artigos científicos, e permite ao investigador uma cobertura mais ampla do número de fenômenos existentes relacionados ao objeto em questão ${ }^{(12)}$.

Nesta pesquisa, optou-se pela investigação de estudos científicos sobre idosos com idade igual ou superior a 80 anos, idoso longevo, ao considerar o atual crescimento de pessoas nessa faixa etária na população idosa, mundial e brasileira, e as peculiaridades sociais que estas demandam.

No primeiro momento do desenvolvimento dessa pesquisa foi realizado o levantamento bibliográfico, no período de agosto a dezembro de 2008, nas bases de dados em ciências da saúde em geral: Scientific Electronic Library Online (SCIELO) e na Literatura Latino-Americana e do Caribe em Ciências da Saúde (LILACS), por meio do acesso à Biblioteca Virtual em Saúde (BVS). O estudo foi subsidiado pelo seguinte critério de inclusão: produções científicas completas, referentes à temática da pessoa idosa longeva (idoso com idade igual ou superior a 80 anos). Foram definidos no projeto de Iniciação Científica como bancos de dados o SCIELO e o LILACS, dos países da América Latina e Caribe, por estas bases possibilitarem o livre acesso a um grande número de publicação eletrônica de periódicos científicos de diversas nacionalidades. Para esta finalidade utilizaram-se como descritores: longevos, octogenários, oitenta anos ou mais, 80 anos ou mais, idosos de oitenta anos ou mais e muito idosos. Após utilizar estes descritores, foram disponibilizados artigos provenientes dos seguintes países: Argentina, Estados Unidos, Peru, Chile, Cuba, México, Espanha e Austrália.

A utilização dessas palavras-chave conduziu a alguns "falso-positivos", ou seja, pesquisas que não eram condizentes com o objeto de estudo. Foram estabelecidos como critérios de exclusão: 1) Artigos que incluíam idosos cuja idade era inferior a 80 anos; 2) Trabalhos não relativos a seres humanos; 3) Trabalhos que não foram conseguidos na íntegra. Assim, foram selecionados apenas os estudos que, com convicção, fazem parte do universo temático investigado.

Esse procedimento possibilitou a identificação de 88 artigos científicos referentes à pessoa idosa longeva, sendo estes publicados nos idiomas português, espanhol e inglês. Para a coleta dos dados foi feito o preenchimento concomitante do instrumento de coleta, através do qual foram observadas as seguintes variáveis independentes: título da obra, autor, objeto de estudo, objetivo, abordagem metodológica, tipo de artigo, área de produção, características geográficas (país onde foi realizado, se Brasil, estado e região), lócus do estudo (instituição pública ou privada), ano de realização, ano e revista de publicação, descritor utilizado e base de dados.

No segundo momento, procedeu-se à análise quantitativa dos dados. Os resultados das variáveis identificadas (base de dados, ano de publicação, tipo de artigo, características geográficas, abordagem metodológica, área de produção e objeto de estudo) foram expressos através de representações numéricas por meio de valores e porcentagens, com gráficos e quadro, seguindo a distribuição sistemática por frequência simples. Este método constitui a estatística descritiva, utilizada para descrever, organizar e sintetizar dados de expressão numérica ${ }^{(13)}$. Posteriormente, os resultados foram discutidos e analisados à luz da produção literária sobre o envelhecimento, produção do conhecimento e longevidade.

\section{RESULTADOS E DISCUSSÃO}

O levantamento bibliográfico utilizando as palavras-chave descritas totalizou 4.381 publicações científicas, das quais 88 $(2 \%)$ do total eram investigações referentes à pessoa idosa longeva. Dessas, constatou-se que $87,5 \%$ foram identificadas na base de dados LILACS.

O singelo percentual de publicações sobre os idosos longevos corrobora os achados de outra pesquisa produzida no ano de 2008, na qual foi feita uma busca na base de dados SCIELO, utilizando as palavras-chave "octogenários" ou "idoso de oitenta anos ou mais", encontrando-se cinco (5) artigos referentes a essa temática ${ }^{(14)}$.

Seguindo a perspectiva do aumento exponencial de octogenários, fenômeno considerado global e evidenciado na realidade brasileira, observa-se a necessidade de investigações científicas nesse universo temático por parte da comunidade acadêmica, já que o resultado encontrado ( $2 \%$ de estudos) comprova a escassez de publicações que abordam os idosos longevos como tema central de pesquisa.

A produção do conhecimento é um processo rápido, dinâmico, que reflete o nível intelectual e profissional de determinada região e momento histórico. Após o desenvolvimento das pesquisas científicas, estas devem ser transmitidas e socializadas ${ }^{(15)}$.

O despertar pela investigação sobre a terceira idade no Brasil vem crescendo a partir da segunda metade do século XX, com aumento considerável na década de 90 . É nesse contexto que a produção de dissertações e teses sobre o processo de envelhecimento humano apresentou um crescimento de forma exponencial a partir da década de 1970, sendo $7 \%$ dos trabalhos concluídos entre os anos de 1975 e 1979; uma terça parte situada nos anos 1980; e mais de $60 \%$ restantes finalizados entre 1990 e $1998^{(16)}$.

Esse interesse se traduziu na constituição de centros de estudo e de formação de profissionais voltados para trabalhar com idosos, na abertura de espaços para reuniões e discussões sobre essa temática, e na criação de áreas especificamente voltadas para o estudo do envelhecimento, no interior de disciplinas acadêmicas plenamente constituídas ${ }^{(17)}$.

De modo geral, o empenho crescente pelo estudo do envelhecimento humano no Brasil coincidiu com o processo de formação dos grupos de pesquisa. O primeiro grupo voltado especificamente para a pesquisa para essa temática foi criado na década de 70, o qual recebeu denominação de NESPI Núcleo de Estudos e Pesquisas do Idoso. Este se constituiu na área de Enfermagem, pautado no estudo da saúde do idoso 
nos aspectos biopsicossociais e instalado na Escola de Enfermagem da Universidade Federal da Bahia. Em 1980, um pequeno número de grupos foi formado, com um aumento considerável destes a partir da década de 1990, período no qual o envelhecimento passa a ser visto também como questão social(16).

No que se refere ao desenvolvimento de pesquisas sobre a população de idosos longevos, este ainda não alcançou números expressivos. Assim, observa-se que, mesmo diante do aumento considerável da expectativa de vida brasileira e mundial, os pesquisadores até o momento não tem dado passos largos na investigação sobre a longevidade.

As publicações sobre a pessoa idosa longeva, de acordo com os resultados desta pesquisa, ocorreram ao longo dos anos de 1994 a 2008 sendo que, nos últimos anos, o despertar por este tema acentuou-se significativamente, constatando-se o maior número de pesquisas (20 artigos), no ano de 2005. Essa distribuição pode ser associada com os períodos de maior discussão sobre o envelhecimento no Brasil, ou seja, quando as leis e políticas públicas foram efetivadas na sociedade brasileira.

Em janeiro de 1994, foi sancionada a LEI No 8.842, que dispõe sobre a Política Nacional do Idoso (PNI), cria o ConseIho Nacional do Idoso e propõe, entre seus princípios e diretrizes, que o envelhecer diz respeito à sociedade em geral, devendo, assim, ser objeto de conhecimento e informação para todos, além de assegurar o apoio a estudos e pesquisas sobre as questões relativas ao envelhecimento ${ }^{(18)}$. Posteriormente, outras medidas foram acrescentadas à criação da PNI, quais sejam: Política Nacional de Saúde do Idoso - Portaria MS 1.395/99, a qual foi atualizada através da Portaria $N^{\circ} 2.528$ em 19 de outubro de 2006 e passou a ser definida como Política Nacional de Saúde da Pessoa Idosa (PNSPI), e a Lei $N^{\circ}$ 10.741 de 1/10/2003, que promulgou o Estatuto do Idoso.

No tocante à distribuição das publicações sobre a pessoa idosa longeva segundo o tipo de artigo, observou-se a predominância de pesquisas classificadas como relato de caso clínico e/ou experiências assistenciais 54(61,4\%), seguido de pesquisa ou original, $22(25 \%)$ do total.

Nesse sentido, fica evidente que a produção do conhecimento relativa a esse tema tem se pautado nos relatos de múltiplas patologias apresentadas pelos idosos longevos, que por sua vez envolvem as doenças crônicas e degenerativas e as síndromes geriátricas, além da descrição de processos terapêuticos e de técnicas cirúrgicas, realizadas nessas pessoas.

Em relação à procedência dos estudos científicos selecionados, podemos analisar que os pesquisadores brasileiros realizaram 69,3\% dos trabalhos sobre a pessoa idosa longeva, seguido do Chile, com a produção de 10,2\% das pesquisas.

Nos anos de 2005-2010, a perspectiva de vida para a população do Brasil correspondia a 72 e 74 anos. Esta se encontrava aquém das apresentadas pela Austrália (81,20 anos), Espanha (80,90 anos), Chile (78,6 anos), Cuba (78,30), EUA (78,20 anos), Argentina (75,30 anos), sendo superior apenas o do Peru $\left(71,40\right.$ anos) ${ }^{(10)}$.

Deste modo, ao avaliar os dados, de acordo com as taxas das expectativas de vida dos países onde os estudos foram desenvolvidos, observou-se que o Brasil se apresenta como predominante nas pesquisas de idosos longevos e, no entanto, não se destaca com o maior índice de esperança de vida, ou seja, com a população mais idosa, quando comparado aos demais países.

O padrão de organização da produção científica no Brasil pôde ser acompanhado nos artigos referentes à pessoa idosa longeva de acordo com as regiões e os estados brasileiros onde foram produzidos, respectivamente.

Pôde-se perceber uma concentração das publicações nas regiões Sudeste $(67,2 \%)$ e Sul $(24,6 \%)$ do país, respectivamente. A região Nordeste apresentou um percentual de $8,2 \%$ das pesquisas, sendo observada ausência destas nas regiões Norte e Centro-Oeste.

Acompanhando o perfil de centralização da investigação científica brasileira, os resultados demonstraram um número elevado de pesquisas sobre idosos longevos no estado de São Paulo, 52,5\%, seguido do Rio Grande do Sul, 23\%. Simultaneamente a estes achados, observou-se que, mesmo sendo com percentuais menores dos encontrados nestes estados, várias unidades da federação brasileira desenvolveram investigações nessa temática.

Nesse campo de investigação, a cidade gaúcha de Veranópolis se destaca com a concentração do desenvolvimento de pesquisas sobre pessoas longevas. Este fato pode ser justificado por esta cidade apresentar uma elevada expectativa de vida ao nascer, 77,7 anos, (na década de 90), enquanto que no Rio Grande do Sul e no Brasil, os índices correspondiam a 72 e 67 anos, respectivamente, na mesma época ${ }^{(19)}$.

A distribuição das produções científicas realizadas no Brasil pode ser compreendida ao considerar a localização não uniforme dos grupos e linhas de pesquisa sobre o envelhecimento humano de acordo com as regiões do país: 59,7\% no Sudeste; $21,5 \%$ no Sul; $13,9 \%$ no Nordeste; $4,9 \%$ no Centro-Oeste, não havendo registro destes na região Norte ${ }^{(16)}$.

Os grupos de pesquisas funcionais pautados no estudo da terceira idade se concentram nas regiões Sudeste e Sul, com maior representatividade nos estados de São Paulo e no Rio Grande do Sul. Esta característica, em algum grau, está relacionada aos programas de pós-graduação em geriatria e gerontologia instalados nestes dois Estados, mais especificamente na Pontifícia Universidade Católica de São Paulo (PUC-SP), na Universidade Estadual de Campinas (Unicamp) e na Pontifícia Universidade Católica do Rio Grande do Sul (PUC-RS) ${ }^{(16)}$.

Nessa perspectiva, os programas de pós-graduação, hoje estabilizados, lato sensu e stricto sensu, em associação com os grupos de pesquisas específicos da investigação do enveIhecimento humano, se caracterizam como espaços oportunos para o desenvolvimento e capacitação de pesquisadores e profissionais especializados na área do idoso, além de possibilitar a produção ativa de conhecimentos nesta temática.

Ao se analisar a abordagem metodológica utilizada nos artigos referentes à pessoa idosa longeva, os resultados apontaram que $77,3 \%$ dos estudos foram realizados com abordagem qualitativa, 12,5\% com abordagem quantitativa, e 10,2\% combinaram os dois métodos. Esse era um achado esperado, pois o maior percentual de publicações sobre idosos longevos 
foi classificado como relato de caso/ experiência $(61,4 \%)$, as quais possuem grande tendência de escolha da análise qualitativa para o desenvolvimento de tais pesquisas.

Esses achados confirmam os resultados apresentados em uma investigação sobre a produção do conhecimento em enfermagem na atenção ao idoso, na qual houve uma concentração de estudos de abordagem qualitativa (50\%), seguidos de $22 \%$ de estudos quantitativos e $6 \%$ de quanti-qualitativos ${ }^{(20)}$.

A adoção do método qualitativo reflete a adaptação deste em aprofundar a compreensão de ações, fenômenos e elementos específicos e delimitáveis, mais pelo grau de complexidade interna do que pela expressão quantitativa desses processos. Esta abordagem adequa-se à execução de estudos de um grupo de indivíduos afetados por determinada doença, não sendo conveniente para a análise de grandes perfis populacionais ou indicadores macroeconômicos e sociais ${ }^{(21)}$.

Quanto ao estudo quantitativo, a sua aplicação, objetiva mostrar grandes números de indicadores, tendências e conjuntos demográficos, através da classificação e transformação destes dados em variáveis compreensíveis. Deste modo, do ponto de vista epistemológico, nenhum dos dois tipos de abordagem metodológica pode ser caracterizado como o mais científico, ao ser comparado com o outro. Assim, a relação entre esses métodos não deve ser refletida como oposição contraditória, uma vez que o estudo quantitativo pode suscitar questões para serem aprofundadas qualitativamente, e vice-versa ${ }^{(21)}$.

As áreas do conhecimento que produziram investigações na temática em estudo foram levantadas e apresentaram a prevalência de artigos realizados por pesquisadores do campo da Medicina $(80,7 \%)$. Além disso, nota-se que as demais áreas pelas quais esses artigos foram desenvolvidos fazem parte da grande área Ciências da Saúde. Este pode ser um viés desse estudo, decorrente da escolha das bases de dados LILACS e SCIELO, as quais são caracterizadas como Ciências da Saúde em geral, e ambas foram acessadas por meio da BVS.

Essas constatações apontam a necessidade de aprofundar os estudos sobre a longevidade nas diversas áreas do conhecimento, adentrando nas ciências humanas e sociais, visto que o envelhecimento populacional interfere diretamente na organização social como um todo.

Foi constatada, também, a mínima proporção de pesquisas que apresentaram pelo menos um pesquisador da Enfermagem, correspondendo a 4,5\% dos estudos. Este é um achado importante, pois evidencia a necessidade dos enfermeiros desenvolverem e divulgarem estudos nesta temática.

A pesquisa em enfermagem ocupa uma posição de destaque, pois autentica o fazer, procura novas formas de cuidar, aproxima as fundamentações teóricas e práticas do exercício da enfermagem, coopera para a manutenção da qualidade de vida da população, produz conhecimento e legitima a prática desses profissionais ${ }^{(15)}$.

O conhecimento produzido pela Enfermagem deve demonstrar, no decorrer do tempo, um compasso ajustado às demandas sociais e às necessidades de enfrentamento da população, especialmente na construção de sua cientificidade, uma vez que esta representa o sustentáculo do cuidar daqueles a quem o enfermeiro dedica o seu mister ${ }^{(1: 60)}$.
A pesquisa em Enfermagem, com foco no processo de envelhecimento humano e nas diferentes repercussões deste fenômeno contemporâneo ainda se encontra em uma fase inicial, quando consideramos outros fenômenos identificados nas práticas de Enfermagem, tais como assistência à mulher e à criança, cuidados clínicos e cirúrgicos, dentre outros, que possuem maior enfoque científico pelos pesquisadores enfermeiros $^{(22)}$.

Para tanto, torna-se essencial a introdução de conteúdos gerontogeriátricos nos currículos de graduação, pós-graduação, especialização e, também, nos programas de educação permanente nos serviços de enfermagem já existentes. Assim, nota-se que a longevidade é acompanhada por demandas peculiares, as quais precisam ser reconhecidas, analisadas e assistidas por profissionais devidamente habilitados ${ }^{(23)}$.

O desenvolvimento de pesquisas sobre o envelhecimento contribui significativamente para a compreensão de diferentes aspectos relacionados às pessoas idosas (determinantes sociais, culturais, biológico), além da sua potencialidade para direcionar o envelhecer com qualidade de vida. Nessa perspectiva, os estudos sobre a pessoa idosa longeva foram analisados na tentativa de identificar e descrever a tendência temática e objetos que vem sendo pesquisados e documentados sobre esse segmento da população. Os objetos mais estudados nas pesquisas estão apresentados na Tabela a seguir.

\section{Tabela1- Categorização dos objetos estudados nas publicações sobre a pessoa idosa longeva. Salvador-BA, 2009.}

\begin{tabular}{lcc}
\hline $\begin{array}{l}\text { Categorias dos objetos de estudo das } \\
\text { publicações sobre pessoa idosa longeva }\end{array}$ & $\mathbf{N}^{\mathbf{0}}$ & $\mathbf{\%}$ \\
\hline $\begin{array}{l}\text { Descrição de casos clínicos e/ou relato de experiência } \\
\text { Patologias: exames diagnósticos, tratamento }\end{array}$ & 70,5 \\
e procedimentos cirúrgicos & 13 & 14,8 \\
Perfil dos longevos e/ou cuidadores & 05 & 5,7 \\
Fator de risco cardiovascular & 04 & 4,5 \\
Fenômeno da longevidade & 04 & 4,5 \\
Total & $\mathbf{8 8}$ & $\mathbf{1 0 0}$ \\
\hline
\end{tabular}

Fonte: dados da pesquisa.

Os resultados evidenciados na Tabela 1 demonstram que $70,5 \%$ das pesquisas relatam situações clínicas que algum idoso longevo apresentou. Ressalta-se que estes trabalhos atingem um número pouco expressivo da população, já que retratam a situação de apenas um indivíduo. Além disso, a maior parte dos relatos de casos clínicos não considerou as peculiaridades da faixa etária desses idosos, tendo um foco singular na doença.

A este percentual somam-se $14,8 \%$ de investigações sobre exames diagnósticos, tratamento e procedimentos cirúrgicos de determinadas patologias, a saber: realização e complicações de cirurgias; procedimentos cardíacos; tratamento da hipertensão; transtornos oculares; prevalência de episódios depressivos e transtorno de ansiedade generalizada nos 
idosos longevos. Ainda nesta perspectiva encontram-se 4,5\% de trabalhos referentes a fatores de risco cardiovascular em octogenários.

Deste modo, nota-se o predomínio de pesquisas envolvendo o idoso longevo no sentido de avaliar algum aspecto relevante a patologias, ou, ainda, a períodos de hospitalização que estes vivenciaram. Poucos pesquisadores investigaram a pessoa idosa longeva a partir de uma abordagem diferenciada da clínico-curativa, fato notado em 5,7\% dos estudos sobre o perfil dos longevos e/ou cuidadores, e 4,5\% de pesquisas referentes ao fenômeno da longevidade. Assim, os resultados desse trabalho confirmam que a população idosa com estado de saúde comprometido, ou com sequelas de enfermidades, permanece sendo o objeto de investigação importante para a realização de pesquisas científicas.

Por meio da análise dos dados observou-se também que, dos 71 trabalhos produzidos na área médica, 69 possuem como foco essencial processos patológicos, enquanto que os quatro artigos publicados pela Enfermagem trazem como enfoque o perfil dos longevos e o processo da longevidade, abordando as dimensões da qualidade de vida e correlações das condições socioeconômicas desses idosos com as práticas de saúde.

A avaliação dos objetos investigados sobre a população de idosos longevos e as lacunas identificadas nas pesquisas referentes a essa temática suscitam a necessidade da produção de conhecimentos sobre a pessoa idosa longeva e as suas peculiaridades.

É preciso considerar que o processo do envelhecimento hoje não é apenas um fenômeno; este se tornou parte da realidade mundial. Assim, diante da real longevidade populacional tem-se como maior desafio colaborar para que os idosos possam redescobrir estratégias de superar os limites que podem advir com o aumento da idade, e viver sua vida com a máxima qualidade possível(6).

O crescimento da população idosa, também caracterizado pelo aumento da longevidade, tende a acarretar maiores índices de doenças crônico-degenerativas, com suas demandas emergentes ou comorbidades comuns a velhice. Somam-se a esses fatores as condições de vida desfavoráveis dos idosos brasileiros, já que estes permanecem sendo vítimas das "enfermidades" sociais, resultante da má distribuição de renda e do retrocesso da economia brasileira ${ }^{(24)}$.

Assim, torna-se visível que o impacto do envelhecimento populacional repercute em toda a sociedade, sobretudo no sistema de saúde, no qual é possível ser observado os déficits relacionados à infraestrutura para atender as demandas da população idosa, referentes à falta de conformação do espaço físico, das políticas, de ações e intervenções específicas e, mesmo, de recursos humanos habilitados para subsidiar as especificidades desse estrato populacional ${ }^{(25)}$.

Os efeitos sociais da transição demográfica e epidemiológica da população lançam como desafio a formulação de uma nova concepção de política pública no Brasil, visto que a longevidade vem associada com a pobreza social. Deste modo, percebe-se que a possibilidade de promover o envelhecer saudável, com independência e autonomia, é o grande objetivo da equipe de saúde. Nesse processo, é imprescindível ter como base os princípios essenciais da universalidade, equidade e integralidade promovidos pelo Sistema Único de Saúde (SUS) ${ }^{(24)}$.

É nessa expectativa que a Política Nacional de Saúde da Pessoa Idosa apresenta, dentre outros desafios, enfrentar a escassez de estruturas de cuidado ao idoso no SUS e de equipes multiprofissionais e interdisciplinares, com conhecimento em envelhecimento e saúde da pessoa idosa. Para tanto tem como uma de suas diretrizes o estímulo e apoio ao desenvolvimento de linhas de pesquisas sobre esse assunto, por meio do estabelecimento de redes de apoio com instituições de ensino e órgãos públicos nas três esferas de governo ${ }^{(26)}$.

Nesse cenário, acredita-se que o alcance do envelhecimento saudável, com autonomia e qualidade, pode ser garantindo através da implantação de políticas e programas, os quais considerem a realidade social, o novo perfil demográfico da população idosa e garanta o incentivo à produção de pesquisas científicas nessa temática. Ademais, vale ressaltar a importância de incluir estratégias capazes de fomentar a ampliação quantitativa e, essencialmente, qualitativa de profissionais capacitados para atuar na área do envelhecimento ${ }^{(27)}$.

\section{CONSIDERAÇÕES FINAIS}

A investigação da produção do conhecimento sobre a pessoa idosa longeva em duas bases de dados das ciências da saúde em geral (SCIELO e LILACS) permitiu-nos vislumbrar o escasso percentual de pesquisas neste universo temático. Este achado suscita a necessidade de estimular os profissionais e pesquisadores a correlacionar os objetos de seus estudos com a nova realidade social: o envelhecimento populacional, seguido do notável aumento global e exponencial do número de idosos longevos na população.

Os resultados dessa pesquisa demonstraram que o Brasil, mesmo não se destacando com o maior índice de expectativa de vida com relação aos demais países aqui englobados, apresenta-se como o país de maior predomínio de estudos sobre a população longeva, com concentração das suas publicações nas regiões Sudeste e Sul, mais especificamente no estado de São Paulo e do Rio Grande do Sul.

Observou-se, ainda, a concentração de trabalhos com enfoque basilar na abordagem clínico-curativa. Houve um número pouco expressivo de estudos referentes ao fenômeno da longevidade e às especificidades dos idosos longevos, com foco na prevenção de doenças e na promoção do envelhecer com bem estar.

Além disso, notou-se que os artigos selecionados de acordo com o objeto deste estudo foram produzidos essencialmente por investigadores da grande área Ciências da Saúde, sem ser identificada a participação de pesquisadores das demais áreas do conhecimento. Quanto à produção de pesquisas pela Enfermagem, notou-se um tímido percentual de investigações referente a essa temática.

Diante dos resultados alcançados, observa-se a necessidade de promover maior visibilidade à pessoa idosa longeva e ao fenômeno da longevidade, considerando as especificidades que emergem nesta etapa da vida. Nesse processo, se torna essencial a formulação e execução de políticas públicas 
sociais e de saúde direcionadas a essa população, bem como o estímulo à produção de conhecimento sobre essa clientela.

Para tanto, são necessários a consolidação e o reconhecimento das particularidades dos idosos longevos por parte das instituições formadoras, universidades e órgãos públicos, de forma que fomentem o avanço científico do tema e a formação de profissionais capacitados e com interesse de fornecer atenção de qualidade a estes cidadãos.

Para o alcance desse objetivo, conclui-se ser fundamental a revisão de currículos de cursos de nível superior e de pós-graduação (Lato e Stricto sensu), das mais diversas áreas do conhecimento, como as Ciências da Saúde, Humanas e Sociais, a fim de que sejam incorporadas a esses espaços reais noções sobre o processo do envelhecimento, aumento da expectativa de vida e as repercussões dessas mudanças no meio social.

Acredita-se que promover transformações nos campos de formação de futuros profissionais, despertando neles o desejo de atenção à população envelhecida, seja a base para o reconhecimento do valor do ser idoso e do envelhecer com qualidade.

\section{REFERÊNCIAS}

1. Moura ERF, Franco ES, Fraga MNO, Damasceno MMC. Produção científica em saúde da mulher na pós-graduação em Enfermagem da Universidade Federal do Ceará, Brasil 1993-2002. Ciencia y enfermeria [periódico online] 2005 [citado 2009 jun14];11(2):59-70. Disponível em: http://www.scielo.cl/scielo.

2. Barros RDB, Castro AM. Terceira idade: o discurso dos experts e a produção do "novo velho". Estud. interdiscip. envelhec. [periódico online] 2002 [citado 2009 mar 10];4:113-124. Disponível em: http://www.seer.ufrgs.br/.

3. Prado SD, Sayd JD. A gerontologia como campo do conhecimento científico: conceito, interesses e projeto político. Ciênc. Saúde Coletiva 2006;11(2):491-501.

4. Santos FH, Andrade VM, Bueno OFA. Envelhecimento: um processo multifatorial. Psicol. estud. 2009;14(1):3-10.

5. Souza RF, Skubs T, Brêtas ACP. Envelhecimento e família: uma nova perspectiva para o cuidado de enfermagem. Rev Bras Enferm. 2007;60(3):263-7.

6. Brasil. Ministério da Saúde. Secretaria de Atenção à Saúde. Departamento de Atenção Básica. Envelhecimento e saúde da pessoa idosa. Brasília: Ministério da Saúde, 2006. Série A. Normas e Manuais Técnicos, Cadernos de Atenção Básica- n. ${ }^{\circ} 19$.

7. Chaimowicz, F. A saúde dos idosos brasileiros às vésperas do século XXI: problemas, projeções e alternativas. Rev. Saúde Públ. 1997;31(2):184-200.

8. Motta LB, Aguiar, AC. Novas competências profissionais em saúde e o envelhecimento populacional brasileiro: integralidade, interdisciplinaridade e intersetorialidade. Ciênc. saúde colet. 2007;12(2):363-72.

9. World Health Organization. Active ageing: a policy framework. Spain: WHO, 2002. 6p.

10. Instituto Brasileiro de Geografia e Estatística (IBGE). Projeções da população do Brasil por sexo e idade 19802050. Rio de Janeiro: IBGE; 2008. Série Estudos e Pesquisas, Informação demográfica e socioeconômica- $n^{\circ} 24$ [citado 2009 fev 14]. Disponível em: http://www.ibge. gov.br/home/estatistica/populacao/projecao_da_populacao/2008/projecao.pdf

11. Gorini MIPC, Severo IM, Silva MCSA. Análise da produção do conhecimento de enfermagem sobre educação em saúde e envelhecimento. Online Brazilian Journal of Nursing [periódico online] 2008 [acessado 2009 jun 08];7(1). Disponível em: http://www.uff.br/objnursing/ index.php/nursing/article/view/j.

12. Gil AC. Como elaborar projeto de pesquisa. 4ed. São Paulo (SP): Atlas; 2006. p.44-5.

13. Polit DF, Beck CT, Hungler BP. Fundamentos de pesquisa em enfermagem: métodos, avaliação e utilização. Tradução de Ana Thorell. 5a. ed. Porto Alegre (RS): Artmed; 2004.

14. Inouye K, Pedrazzani ES, Pavarini SCl. Octogenários e cuidadores: perfil sócio-demográfico e correlação da variável qualidade de vida. Texto Contexto Enferm. 2008;17(2):350-7.

15. Costa RS, Carvalho DV. Análise da produção científica dos enfermeiros de Minas Gerais publicada em periódicos de enfermagem. Rev Latino-Am. Enfermagem 2001;9(5):19-25.

16. Prado SD, Sayd JD. A pesquisa sobre envelhecimento humano no Brasil: grupos e linhas de pesquisa. Ciênc. saúde colet. 2004;9(1):57-68.

17. Neri AL, Debert GG (Orgs). Velhice e sociedade. Campinas (SP): Papirus; 1999.

18. Ministério da Saúde (BR). Lei $\mathrm{n}^{\circ} 8.842$, de 4 de janeiro de 1994. Dispõe sobre a política nacional do idoso, cria o Conselho Nacional do Idoso e dá outras providências. Diário Oficial da União 1994; jan 5. Disponível em: http:// www.sbgg.org.br/profissional/legislacao/lei_idoso.pdf.

19. Cruz IBM, Almeida MSC, Schwanke CHA, Moriguchi EH. Prevalência de obesidade em idosos longevos e sua associação com fatores de risco e morbidades cardiovasculares. Rev. Assoc. Med. Bras. [periódico online] 2004 [citado 2008 out 16];50(2):172-177. Disponível em: http:// www.scielo.br/scielo.php.

20. Veiga KCG, Menezes TMO. Produção do conhecimento em enfermagem: a (in) visibilidade da atenção à saúde do idoso. Rev Esc Enferm USP 2008; 42(4): 761-8.

21. Minayo MCS, Sanches O. Quantitativo-qualitativo: oposição ou complementaridade? Cad. Saúde Públ. 1993;9(3):237-48.

22. Rocha FCV, Brito CMS, Luz MHBA, Figueiredo MLF. Análise da produção científica sobre o idoso na REBEn. Rev Bras Enferm 2007;60(4):449-51.

23. Diogo MJD, Duarte YAO. O envelhecimento e o idoso de graduação em enfermagem no Brasil: do panorama 
atual a uma proposta de conteúdo programático. Rev Esc Enferm USP 1999;33(4):370-6.

24. Gonçalves LHT, Schier J. "Grupo aqui e agora" uma tecnologia leve de ação sócio-educativa de enfermagem. Texto Contexto Enferm 2005; 14(2):271-9.

25. Leite MT, Gonçalves LHT. A enfermagem construindo significados a partir de sua interação social com idosos hospitalizados. Texto Contexto Enferm 2009;8(1):108-15.
26. Ministério da Saúde (BR). Portaria $\mathrm{n}^{\circ} 2.528$ de 19 de outubro de 2006. Aprova a Política Nacional de Saúde da Pessoa Idosa. Diário Oficial da União 2006 out 20. Saúde do Idoso/Legislação. Brasília, 2006 [citado 2009 jul 18]. Disponível em: http://portal.saude.gov.br/portal/saude/visualizar_texto.cfm.

27. Motta LB, Caldas CP, Assis M. A formação de profissionais para a atenção integral à saúde do idoso: a experiência interdisciplinar do NAI - UNATI/UERJ. Ciênc. saúde colet. 2008;13(4):1143-51. 


\section{ERRATAS}

Vol.64 № 4 jul./ago. 2011

\begin{tabular}{|c|c|c|}
\hline PÁGINA & ONDE SE LÊ & LEIA-SE \\
\hline 751 & Talita Aquira Santos Lima & Talita Aquira dos Santos Lima \\
\hline 751 & $\begin{array}{l}\text { Conclui-se que a produção científica sobre o idoso } \\
\text { longevo ainda é singela e irrelevante. }\end{array}$ & $\begin{array}{l}\text { Conclui-se que a produção científica sobre o idoso } \\
\text { longevo ainda é singela, mas relevante. }\end{array}$ \\
\hline 751 & $\begin{array}{l}\text { One concluded that the scientific production on } \\
\text { long-lived elderly person is still small and irrelevant. }\end{array}$ & $\begin{array}{l}\text { One concluded that the scientific production on } \\
\text { long-lived elderly person is still small, but relevant. }\end{array}$ \\
\hline 751 & $\begin{array}{l}\text { Se concluye que la producción científica sobre el } \\
\text { mayor de } 80 \text { anos es todavía aun simple e irrelevante. }\end{array}$ & $\begin{array}{l}\text { Se concluye que la producción científica sobre el } \\
\text { mayor de } 80 \text { anos es todavía aun simple, más relevante. }\end{array}$ \\
\hline 751 & $\begin{array}{l}\text { AUTOR CORRESPONDENTE } \\
\text { Talita Aquira Santos Lima talyaquira@gmail.com }\end{array}$ & $\begin{array}{l}\text { AUTOR CORRESPONDENTE } \\
\text { Talita Aquira dos Santos Lima talyaquira@hotmail.com }\end{array}$ \\
\hline \multicolumn{3}{|c|}{ Vol.64 № 5 set./out. 2011} \\
\hline PÁGINA & ONDE SE LÊ & LEIA-SE \\
\hline 912 & $\begin{array}{l}\text { Extraído de Pesquisa de Iniciação Científica, financiada } \\
\text { pela FAPEMIG, intitulada "Dimensionamento de pessoal de } \\
\text { enfermagem frente à classificação dos clientes hospitalizados } \\
\text { em um hospital de ensino". }\end{array}$ & $\begin{array}{l}\text { Extraído da Dissertação "Práticas alimentares no primeiro } \\
\text { ano de vida na percepção de mães adolescentes", Programa } \\
\text { de Pós-Graduação em Saúde da Criança e do Adolescente, } \\
\text { Universidade Federal de Pernambuco, } 2011 .\end{array}$ \\
\hline
\end{tabular}

